\title{
Outpatient follow-up in women with HIV infection in Parkside Health Authority (UK)
}

\author{
P J Horner, M McBride, R J Coker, S Crowley, J R W Harris, S M Murphy, J N Weber, \\ A M Renton
}

\begin{abstract}
Objective-To describe patterns of attendance for follow-up among HIV infected women in Parkside, UK and their correlates.

Design-Restrospective cohort study. Subjects-103 HIV infected women.

Main outcome measures-Whether patients attended for follow-up between three and 18 months.

Results-31\% of women were married and $46 \%$ had children. Women born in sub-Saharan Africa were significantly less likely to attend for follow-up after three months (56\%) than women born in other areas who had acquired HIV either heterosexually $(82 \%)$ or through injecting drug use $(81 \%)$. This pattern persisted on multivariate analysis controlling for whether women were symptomatic, had had a previous positive test, were married or had children.

Conclusions-HIV positive sub-Saharan African women are less likely to reattend for follow-up than women with heterosexually acquired HIV from other areas or those who acquired infection through intravenous drug use. Further studies are needed to identify barriers to follow-up for women and to shape the development of more appropriate and accessible services for HIV infected women, especially those of sub-Saharan African origin.
\end{abstract}

(Genitourin Med 1993;69:370-372)

Genitourinary

Medicine and

Communicable

Diseases, St Mary's

Hospital, London W2

INY, UK

P J Horner

M McBride

R J Coker

$S$ Crowley

$J R$ W Harris

J N Weber

Department of

Genitourinary

Medicine, Patrick

Clements Clinic,

Central Middlesex

Hospital, London

NW10 7NS, UK

$S$ M Murphy

Academic Dept of

Public Health, St

Mary's Hospital

Medical School,

London W2 1NY, UK

A M Renton

Address correspondence to: Dr PJ Horner

Accepted for publication 26 May 1993 improving the health of the nation. The of the White Paper is to match gets with a strategy through which they might be achieved. The recommendations of the national Health of the Nation focus group on
HIV/AIDS and Sexual Health ${ }^{6}$ give particular emphasis to the importance of providing accessible services which match the particular needs of different local groups.

Regular follow-up of HIV infected individuals is important in achieving adequate clinical management and health promotion and may be particularly important in women of reproductive age. Clinical monitoring of HIV infected patients has been shown to be beneficial in reducing the associated morbidity and earlier mortality. ${ }^{78}$ It has been suggested that patients of sub-Saharan African origin may be less likely to attend for follow-up. We reviewed our experience with HIV infected women in Parkside District Health Authority to examine the patterns and determinants of attendance for follow-up.

\section{Patients and methods}

One hundred and three women who presented to the Jefferiss Wing Genitourinary medicine (GUM) clinic or to the Patrick Clements GUM clinic in Parkside Health Authority between March 1986 and March 1991 were identified. All these women tested positive for HIV antibodies at presentation or were known to be HIV antibody positive by prior test. This represented a complete sample of such women identified by the two clinic registers.

Between June and December 1992 clinical records were obtained for all patients. Information was collected on country of birth, age, history of risk behaviour and HIV1 antibody tests, Center for Disease Control (CDC) stage of disease, ${ }^{9}$ marital status, and whether or not the woman had children. The most likely mode of HIV-1 transmission was identified from the history of risk behaviour documented at the time of HIV testing. Information on country of birth was not available in one patient. In addition it was noted whether the patient had attended at any time between 90 days and 546 days subsequent to first presentation. Where this was the case they were classified as being in follow-up between three and 18 months. In all cases patient notes were viewed at least 18 months after the patient had presented.

Women were divided into three study groups for the purposes of analysis: women born in sub-Saharan Africa who had acquired HIV infection heterosexually (SSA), women born outside sub-Saharan Africa who had acquired HIV infection heterosexually (Non SSA), and women born outside sub-Saharan 
Africa who had acquired HIV infection through injecting drug use (IDU).

Women classified as CDC disease stage IV were designated as having symptomatic HIV infection. The remaining women were designated as not having symptomatic HIV infection.

Data analyses were performed using SAS and EGRET statistical software. Chi square has been used throughout to test the differences between proportions. Logistic regression was used for the multivariate analysis.

\section{Results}

Of the 103 women, 74 (72\%) attended for follow-up between 3 and 18 months. The median age was 26 years. Thirty two (31\%) were married, and $47(46 \%)$ had children. Thirteen (13\%) had symptomatic HIV infection, and 47 (46\%) had had an HIV test prior to presentation. Forty (39\%) were subSaharan African, 28 (27\%) were non subSaharan African who had acquired HIV heterosexually, and 34 (33\%) had acquired HIV through injecting drug use. We examined the relationship between the characteristics of women and follow-up attendance (table 1).

The proportion of sub-Saharan African women attending for follow-up between three and 18 months was significantly smaller than that among both non sub-Saharan African women who had acquired infection heterosexually and women who had acquired infection through injecting drugs $(p<0.03)$. Married women were less likely to attend for follow-up, although the difference did not

Table 1 Follow-up attendance by features of subjects

\begin{tabular}{lll}
\hline & $N$ & $\begin{array}{l}\text { No. (\%) in follow-up } \\
\text { (18 months }\end{array}$ \\
\hline Group: & & \\
SSA & 40 & $23(57)$ \\
Non SSA & 28 & $23(82)$ \\
IDU & 34 & $28(83)$ \\
Married: & 32 & $19(59)$ \\
$\quad$ Yes & 71 & $55(77)$ \\
$\quad$ No & 47 & $33(70)$ \\
Children: & 55 & $40(73)$ \\
$\quad$ Yes & 13 & $11(85)$ \\
No & 90 & $63(70)$ \\
Symptoms of HIV: & & $34(72)$ \\
$\quad$ Yes & 47 & $40(72)$ \\
No & 56 & \\
Previous HIV test: & Yes &
\end{tabular}

Table 2 Features of subjects by study group

\begin{tabular}{lllr}
\hline & $\begin{array}{l}\text { Sub Saharan } \\
\text { African (SSA) }\end{array}$ & $\begin{array}{l}\text { Non SSAI } \\
\text { non IDU }\end{array}$ & IDU \\
\hline No. of subjects & 40 & 28 & 34 \\
Median age (years) & 26 & 31 & 27 \\
No. (\%) married & $18(45)$ & $9(32)$ & $4(12)$ \\
No. (\%) with children & $26(65)$ & $11(41)$ & $9(26)$ \\
No. (\%) with HIV associated & $4(10)$ & $4(14)$ & $5(14)$ \\
symptoms & $9(23)$ & $14(50)$ & $23(68)$ \\
No. (\%) with previous HIV test & & & \\
\hline
\end{tabular}

reach significance $(p=0.098)$. There were no differences between symptomatic and asymptomatic women or between those with and without children in proportions followed up. The median age for both attenders and non-attenders for follow-up was 26 years.

We compared the characteristics of women between the three study groups (table 2). Sub-Saharan African women were four times more likely to be married ( $p<0.005$; chi square $[1 \mathrm{df}]=8 \cdot 19)$ and more than twice as likely to have children ( $p<0.005$; chi square [1df $=9.45)$ than women who had acquired infection through injecting drug use. They were also less likely to have had an HIV test prior to presentation at the clinic than both the non sub-Saharan African women who had heterosexually acquired HIV ( $p<0.05$; chi square $[1 \mathrm{df}]=4 \cdot 40$ ) and the non SubSaharan African group who acquired HIV through injecting drug use $(p<0.001$; chi square $[1 \mathrm{df}]=13 \cdot 48)$. There were no other significant differences between the three groups in the parameters recorded (table 2).

In order to assess the influence of child care and other domestic responsibilities, disease stage, and prior positive HIV antibody test on the differential follow-up rates, we carried out a multivariate analysis by logistic regression using attendance as the outcome variable. In addition to study group, we included the variables for marital status, whether or not the women had children or symptoms, and prior positive HIV antibody test as independent terms in the model. For the patient groups odds ratios for attendance were calculated against baseline attendance odds for the sub-Saharan African group. Both the non sub-Saharan group who acquired HIV heterosexually and the group who acquired HIV through injecting drug use were significantly more likely to attend than the sub-Saharan African group; for the non sub-Saharan group OR $=3.81$ (95\% CI: 1.08 13.4, p < 0.04), and for those with HIV acquired through injecting drug use $\mathrm{OR}=$ 3.79 (95\% CI:1.00-14.3, p = 0.05). No other variable was significantly related to attendance.

\section{Discussion}

We have reported follow-up and characteristics of all women who were known or diagnosed to be infected with HIV when they presented to two Parkside Genitourinary Medicine clinics between March 1986 and March 1991. Overall $28 \%$ of women did not attend for follow-up at any time during the period studied. Only $52 \%$ of women of subSaharan African origin attended the clinics during the follow-up period, compared with $82 \%$ of non sub-Saharan African women who had acquired infection heterosexually and $83 \%$ of non sub-Saharan African women who had acquired infection through injecting drug use. Although sub-Saharan African women were more likely to be married and to have children, and less likely to have had a prior positive HIV test than the other groups, these 
did not explain the differences in follow-up observed between the groups.

Recent evidence suggests that the provision of health services for HIV seropositive patients may be poorly geared for women. ${ }^{1011}$ Our findings emphasise the substantial proportions of HIV antibody positive women who are married $(31 \%)$ and who have children $(46 \%)$, although neither these factors, nor having symptoms of HIV infection or a prior positive HIV antibody test seem to influence attendance for follow-up. Women attending a genitourinary medicine clinic in London have expressed a preference for an all-female staffed multidisciplinary outpatient service. ${ }^{10}$ Experience from one such service suggests that women diagnosed HIV antibody positive at the all-woman clinic show significantly higher rates of follow-up than those achieved among women diagnosed positive at a general GUM clinic. ${ }^{11} \mathrm{HIV}$ antibody positive women have particular requirements for health care services which differ from those of HIV positive homosexual men; for example, obstetric, gynaecological and family planning services, the provision of creche facilities for children, and health care for those children that may also be infected with HIV.

We cannot say from this study why the poor rate of follow-up in sub-Saharan female patients should have occurred. Explanations may include cultural barriers, attendance at other clinics or travel abroad, greater preference for all-female staff, language difficulties, or greater social and work commitments. In particular, problems in the availability of medical care in patients' country of origin may lead to quite different perceptions of the degree of extremity of illness at which it is appropriate to seek medical care. Furthermore, the benefits of seeking care early may not be adequately appreciated.

We are concerned that HIV infected women may not be gaining access to appropriate health care. Greater efforts are required to ensure that appropriate messages and information about potential benefit of regular care are given during pre and post test counselling. In addition further studies are required to identify women's needs and the barriers to their attendance which will help us define the shape of more accessible and appropriate services provisions for these women. Given the increasing number of women who are identified as HIV antibody positive who are from sub-Saharan Africa, a particular focus on the needs of this group is urgently needed.

1 Evans BG, Noone A, Mortimer JY, et al. Heterosexually acquired HIV-1 infection: cases reported in England Wales and Northern Ireland, 1985 to 1991. Communicable Disease Report 1992;2:R49-55.

2 Mitchell S, Band B, Bradbeer C, Barlow D. Imported Heterosexual HIV infection in London. Lancet 1991; Heterosexual

3 O'Farrell N, Allen M, Gardner F. Imported Heterosexual HIV infection. Lancet 1991;338:386-7.

4 Chrystie II, Palmer SJ, Kenney A, Banntvala JE. HIV seroprevalence among women attending antenata clinics in London. Lancet 1992;339:364.

5 Noone A, Gill ON, Clarke SE, Porter K. Travel, heterosexual intercourse and HIV-1 infection. Communicable Disease Report 1991;1:R39-43.

6 Secretary of State for Health. The Health of the Nation: a Strategy for Health in England. London HMSO, 1992. (CM 1986.)

7 Brettle RP, Leen CLS. The natural history of HIV and AIDS in women. AIDS 1991;5:1283-92.

8 Carpenter CJ, Mayer KH, Stein MD, et al. Human immunodeficiency virus infection in North American women: Experience with 200 cases and a review of the literature (Baltimore) Medicine 1991;70:307-25.

9 Revision of the CDC surveillance case definition for acquired immunodeficiency syndrome. MMWR 1987; 36(suppl 1S): S1-15.

10 Kell PD, Barton SE, Boag FC. Incorporating patients views in planning services for women with HIV infection. Genitourin Med 1992;68:233-4.

11 McCarthy GA, Cockell AP, Kell PD, Beevor AS, Boag FC. A women only clinic for HIV, genitourinary medicine and substance misuse. Genitourin Med 1992;68: 386-9. 